\title{
Associação entre privação do sono e obesidade em trabalhadores
}

\author{
Association between sleep deprivation and obesity in workers
}

\author{
Laís Benvegnú ${ }^{1}$, Marina Maffessoni ${ }^{1}$, Simone Pereira Fernandes ${ }^{2}$, Raquel Canuto ${ }^{3} \bowtie$ \\ 1 Instituto de Ensino, Pesquisa e Gestão em Saúde (IPGS). Porto Alegre, RS. \\ 2 Programa de Pós-Graduação em Saúde da Criança e do Adolescente da Universidade Federal do Rio Grande do Sul. Porto Alegre, RS \\ ${ }^{3}$ Departamento de Nutrição da Universidade Federal do Rio Grande do Sul. Porto Alegre, RS.
}

\section{RESUMO}

Objetivos: estudar a associação entre privação de sono e obesidade em trabalhadores.

Métodos: Foi conduzido um estudo transversal com trabalhadores de uma manufatura de cadernos no sul do Brasil, que possui três setores de trabalho (linha de produção, setor administrativo e logística), sendo que o setor de produção trabalha 24 horas por dia. Características demográficas, socioeconômicas, ocupacionais e comportamentais foram obtidas através de um questionário autopreenchível, padronizado e pré-testado. As medidas de peso e altura foram autorreferidas e o índice de massa corporal calculado. O ponto de corte para obesidade foi de $\geq 30 \mathrm{~kg} / \mathrm{m}^{2}$. A duração do sono foi categorizada em no mínimo seis horas por dia e menos de seis horas por dia (privação do sono). Para análise bivariada, foi utilizado o teste do Qui-quadrado e o teste de tendência linear. As razões de prevalência brutas e ajustadas foram calculadas pela Regressão de Poisson.

Resultados: A amostra final contou com 193 trabalhadores, com média de idade de 34 111 anos, sendo 99 (51\%) mulheres. A prevalência de obesidade foi de $27(13,98 \%)$, e a prevalência de privação do sono foi de 40 (20,7\%). A privação de sono foi associada ao setor de produção $(\mathrm{p}=0,011)$, ao turno noturno $(\mathrm{p}<0,001)$ e à inatividade física $(\mathrm{p}=0,003)$. A presença de obesidade foi associada à privação do sono $(\mathrm{p}=0,006)$. Após o controle para possíveis fatores de confusão, a probabilidade de ser obeso foi de 2,37 (IC 95\% 1,08-5,21) vezes maior entre trabalhadores em privação de sono.

Conclusões: Os resultados demostraram associação entre privação de sono e obesidade. Além disso, a privação de sono foi associada ao trabalho no turno noturno.

DESCRITORES: obesidade; sono; doenças profissionais.

\section{ABSTRACT}

Aims: To study the association between sleep deprivation and obesity among workers.

Methods: We conducted a cross-sectional study of workers at a notebooks manufacturing in southern Brazil, which has three sectors of work (production line, back office and logistics), and the production sector works 24 hours a day. Demographic, socioeconomic, occupational and behavioral characteristics were obtained by a self-completion questionnaire, standardized and pre-tested. Weight and height measurements were self-reported and body mass index calculated. The cutoff point for obesity was $\geq 30 \mathrm{~kg} / \mathrm{m}^{2}$. Sleep duration was categorized into at least six hours a day and less than six hours a day (sleep deprivation). For bivariate analysis, chi-square test and test for linear trend were used. The crude and adjusted odds ratios were calculated using Poisson regression.

Results: The final sample consisted of 193 workers, with a mean age of $34 \pm 11$ years, $99(51 \%)$ women. The prevalence of obesity was $27(13.98 \%)$, and the prevalence of sleep deprivation was $40(20.7 \%)$. Sleep deprivation has been associated with the production sector $(\mathrm{p}=0.011)$, the night shift $(\mathrm{p}<0.001)$ and physical inactivity $(\mathrm{p}=0.003)$. The presence of obesity was associated with sleep deprivation $(\mathrm{p}=0.006)$. After controlling for potential confounding factors, the likelihood of being obese was 2.37 (95\% CI 1.08 to 5.21) times higher among workers in sleep deprivation.

Conclusions: The results showed an association between sleep deprivation and obesity. In addition, sleep deprivation was associated with work on the night shift.

KEY WORDS: obesity; sleep; occupational diseases 
Abreviaturas: VIGITEL, vigilância de fatores de risco e proteção para doenças crônicas por inquérito telefônico; IMC, índice de massa corporal; RP, razão de prevalência; IC, intervalo de confiança; NHANES I, National Health and Nutrition Examination I

\section{INTRODUÇÃO}

A obesidade é um grande problema de saúde pública, que afeta cerca de 450 milhões de pessoas em todo o mundo [1] e, com isso, tem contribuído para o aumento da morbimortalidade populacional [2,3]. Estudos têm demostrando que o excesso de peso está associado à hipertensão [4,5], apneia obstrutiva do sono $[4,6]$, intolerância à glicose, diabetes mellitus tipo II [7,8], câncer [9], doença vascular cerebral e cardiopatias $[10,11]$. No Brasil, em 2014, o estudo de Vigilância de Fatores de Risco e Proteção para Doenças Crônicas por Inquérito Telefônico (VIGITEL) demonstrou que $52,5 \%$ dos brasileiros apresentavam excesso de peso e 17,9\% apresentavam obesidade [12].

A literatura científica recente tem apontado as características laborais, como longas jornadas de trabalho realizadas no esquema de trabalho por turnos, como importante fator de risco para aumento da prevalência de obesidade [13-16]. O trabalho por turnos compreende escalas de trabalho diferentes das oito horas diárias usuais de trabalho, que incluem trabalho no turno da noite, trabalho por turnos rotativos (nas três fases das 24 horas do dia) e trabalho com horários irregulares ou alternativos [17]. Atualmente, muitas empresas e instituições estão se movendo em direção a um padrão de funcionamento de 24 horas por dia. Dessa forma, uma quantidade crescente de indivíduos submete-se ao trabalho por turnos, o que leva a mudanças gerais de comportamento e rotina, incluindo restrição de horas de sono [15,16,18-20].

A quantidade diária adequada de sono e o sincronismo do ritmo circadiano são necessários para atingir um equilíbrio no balanço energético e consequentemente contribuir na regulação do peso corporal [21,22]. A privação do sono está associada a várias consequências adversas, como aumento do apetite [23], comprometimento da sensibilidade à insulina [24-26] e aumento dos níveis de colesterol total e de lipoproteína de baixa densidade [27], culminando em distúrbios metabólicos [18,28,29] e doenças cardiovasculares [30-33].

Nesse sentido, os trabalhadores de turnos seriam mais expostos à privação de sono e, consequentemente, aos distúrbios metabólicos, como a obesidade. Ainda que nos países da América do Sul exista um rápido crescimento da massa laboral admitida em empresas que funcionam 24 horas por dia, em esquemas de turnos, após revisão da literatura nas principais bases de dados internacionais foram localizados poucos estudos que investigaram as consequências da privação de sono sobre a obesidade nessa população $[15,16,26]$. Assim, o objetivo desse estudo foi investigar a associação entre a privação de sono e a obesidade em trabalhadores que exercem suas atividades laborais em uma empresa que funciona nas 24 horas do dia.

\section{MÉTODOS}

Foi realizado um estudo transversal, com trabalhadores de ambos os sexos, com idade entre 18 e 60 anos, de uma empresa de manufatura de cadernos localizada no interior do Rio Grande do Sul, Brasil, que funciona nas 24 horas do dia. Foram incluídos no estudo trabalhadores que tinham contrato de trabalho há pelo menos três meses e que aceitaram participar da pesquisa. Mulheres grávidas e trabalhadores afastados da empresa há mais de 15 dias ou em férias no período da coleta dos dados não foram incluídos.

A coleta das informações ocorreu durante 45 dias, entre os meses de março e abril de 2015, por meio de um questionário autopreenchível, padronizado e pré-testado. Cada participante levou um questionário para casa, com prazo de retorno de sete dias. Foram excluídos os participantes que não devolveram os questionários e aqueles cujas respostas não continham todas as informações necessárias.

O projeto foi aprovado pelo Comitê de Ética da Universidade Federal do Rio Grande do Sul, sob o número de protocolo 967.768 .

$\mathrm{O}$ peso e a estatura foram autorrelatados. A obesidade foi categorizada pelo índice de massa corporal (IMC), obtido pela relação entre peso (quilogramas) e estatura (metros) ao quadrado. $\mathrm{O}$ ponto de corte para obesidade foi IMC $\geq 30 \mathrm{~kg} / \mathrm{m}^{2}$ [34].

As variáveis sociodemográficas investigadas foram sexo (masculino e feminino); idade (em quintis); cor da pele (branca ou outra); estado civil (com companheiro e sem companheiro); escolaridade (ensino fundamental incompleto/completo, até oito anos de estudo; ensino médio incompleto/completo, nove a 11 anos de estudo; e ensino superior incompleto/ completo, graduação em andamento ou concluída); e renda familiar (menos de três salários mínimos, de três a cinco salários mínimos e mais de cinco salários mínimos). 
A obesidade dos pais foi subjetivamente estimada pelo respondente e categorizada em: "nenhum dos pais obeso", "pai ou mãe obeso", "pai e mãe obesos". As variáveis comportamentais foram: atividade física de lazer (nunca, três ou menos vezes por semana e mais de três vezes por semana), número de refeições por dia (duas refeições ou menos, três refeições, quatro ou mais refeições), tabagismo (não fumante e fumante), uso de álcool (nunca, menos de uma vez por semana $\mathrm{e}$ uma vez ou mais por semana).

A empresa funcionava de segunda a sábado e possuía três setores de trabalho: linha de produção, setor administrativo e logística. Somente o setor de produção funcionava por 24 horas diárias. Os turnos tinham duração que variava de acordo com a tarefa desempenhada, com os seguintes horários de começo e término: turno 1 , entrada às $0 \mathrm{~h}$ e saída às $6 \mathrm{~h}, 7 \mathrm{~h}$ ou $8 \mathrm{~h} 30 \mathrm{~min}$; turno 2 , entrada às $6 \mathrm{~h}, 7 \mathrm{~h} 20 \mathrm{~min}$ ou $8 \mathrm{~h} 20 \mathrm{~min}$, saída às $14 \mathrm{~h} 40 \mathrm{~min}$; turno 3 , entrada às $14 \mathrm{~h} 40 \mathrm{~min} \mathrm{e}$ saída às $19 \mathrm{~h} 20 \mathrm{~min}, 20 \mathrm{~h} 20 \mathrm{~min}$ ou $24 \mathrm{~h}$. Jornadas com mais de seis horas tinham pausa de uma hora para alimentação e descanso. Os trabalhadores eram fixos no setor e horário. Para as análises deste estudo, foram considerados trabalhadores noturnos os trabalhadores com horário de trabalho entre $0 \mathrm{~h}$ e $8 \mathrm{~h} 30 \mathrm{~min}$ (turno 1). Os demais foram classificados como trabalhadores diurnos (turnos 2 e 3 ).

Para avaliar a duração do sono, foram coletadas informações sobre o horário em que o trabalhador dormia e acordava habitualmente e se ele realizava algum cochilo adicional durante o dia. Após, foram contabilizadas as horas de sono, categorizadas em menos de seis horas por dia e seis horas ou mais por dia. Considerou-se menos de seis horas diárias como privação de sono. Foi também questionado o consumo de medicamentos para dormir.

A análise dos dados foi feita nos programas IBM SPSS Statistics 20 e Stata 12. As variáveis foram descritas por meio de frequências absolutas e relativas. Para análise bivariada, foi utilizado o teste do Qui-quadrado para variáveis dicotômicas e teste de tendência linear para varáveis ordinais. As razões de prevalência brutas e ajustadas foram calculadas pela Regressão de Poisson e o teste de Wald foi utilizado para testar a significância de cada variável do modelo. A análise seguiu modelo conceitual de análise $a$ priori. No modelo 1 não houve ajustes. O modelo 2 foi ajustado para as variáveis sociodemográficas. O modelo 3 para o modelo 2 para as variáveis laborais. O modelo 4 para os modelos 2 e 3 e para as variáveis comportamentais. Foram incluídas e retidas nos modelos, como potenciais fatores de confusão, as variáveis que apresentassem $\mathrm{p}<0,20$ na análise bivariada. O nível de significância adotado foi de $5 \%(\mathrm{p} \leq 0,05)$.

\section{RESULTADOS}

No momento da coleta de dados a empresa empregava 465 trabalhadores, distribuídos nos três setores de trabalho (linha de produção, setor administrativo e logística). Setenta e quatro trabalhadores estavam de férias. Todos os outros 391 trabalhadores eram elegíveis e foram convidados a participar da pesquisa no momento da entrada, saída e intervalo do trabalho. Destes, 257 trabalhadores aceitaram participar da pesquisa, assinaram o Termo de Consentimento Livre e Esclarecido e receberam os questionários. Vinte e seis questionários não foram devolvidos e 38 foram excluídos por dados faltantes. Assim, a amostra final contou com 193 trabalhadores.

A média de idade dos participantes foi de $34 \pm 11$ anos e 99 (51\%) eram mulheres. A prevalência de trabalhadores com obesidade foi de 27 (13,98\%). A prevalência de trabalhadores em privação do sono, caracterizada por menos de seis horas de sono por dia, foi de $40(20,7 \%)$.

A Tabela 1 descreve a prevalência de privação de sono de acordo com características demográficas, socioeconômicas, ocupacionais e comportamentais dos trabalhadores. Maiores prevalências de privação do sono foram observadas entre os trabalhadores do setor da produção e que relataram não praticar atividades físicas. Na análise bivariada, a prevalência de privação do sono foi quatro vezes maior entre os trabalhadores do turno noturno, quando comparados aos do turno diurno.

Na Tabela 2 pode-se observar a distribuição da prevalência de obesidade de acordo com as variáveis explanatórias. A obesidade foi mais prevalente em pessoas com idade acima de 43 anos e com baixa escolaridade, além ser mais prevalente em trabalhadores que tinham o hábito de dormir menos de seis horas por dia.

A Tabela 3 mostra as razões de prevalência (RP) brutas e ajustadas e intervalos de confiança (IC) $95 \%$ para associação entre privação do sono e obesidade. Os trabalhadores em privação de sono tiveram uma probabilidade 2,37 vezes maior de desenvolver obesidade (RP 2,37; IC95\% 1,08-5,21), quando comparados aos que dormiam seis horas ou mais, após ajuste nos três modelos. Ao inserir as variáveis sociodemográficas (modelo 1), a associação entre privação do sono e obesidade perdeu significância estatística. Porém, ao inserir as variáveis comportamentais (modelo 3 ) a associação voltou a ser significativa. 
Tabela 1. Descrição da amostra e prevalência de privação de sono (menos de seis horas de sono por dia) de acordo com características demográficas, socioeconômicas, ocupacionais e comportamentais de uma população de trabalhadores de uma manufatura do sul do Brasil, 2015.

\begin{tabular}{|c|c|c|c|}
\hline Variáveis & n (\%) & $\begin{array}{r}\text { Privação } \\
\text { do sono }\end{array}$ & p \\
\hline $\begin{array}{l}\text { Sexo } \\
\text { Feminino } \\
\text { Masculino }\end{array}$ & $\begin{array}{l}99(51,3) \\
94(48,7)\end{array}$ & $\begin{array}{l}21,2 \% \\
20,2 \%\end{array}$ & $0,864 *$ \\
\hline $\begin{array}{l}\text { Idade (quartis) } \\
\qquad \begin{array}{l}18 \text { a } 25 \text { anos } \\
26 \text { a } 30 \text { anos } \\
31 \text { a } 42 \text { anos } \\
43 \text { a } 60 \text { anos }\end{array}\end{array}$ & $\begin{array}{l}47(24,4) \\
48(24,9) \\
50(25,9) \\
48(24,9)\end{array}$ & $\begin{array}{l}25,5 \% \\
14,6 \% \\
18,0 \% \\
25,0 \%\end{array}$ & $0,939^{+}$ \\
\hline $\begin{array}{l}\text { Cor de pele } \\
\text { Branco } \\
\text { Não branco }\end{array}$ & $\begin{array}{c}156(80,8) \\
37(19,2)\end{array}$ & $\begin{array}{l}19,9 \% \\
24,3 \%\end{array}$ & $0,548^{*}$ \\
\hline $\begin{array}{l}\text { Estado civil } \\
\text { Com companheiro } \\
\text { Sem companheiro }\end{array}$ & $\begin{array}{c}135(69,9) \\
58(30,1)\end{array}$ & $\begin{array}{l}20,7 \% \\
20,7 \%\end{array}$ & $0,994^{*}$ \\
\hline $\begin{array}{l}\text { Escolaridade } \\
\text { Ensino fundamental } \\
\text { ( } \leq 8 \text { anos de estudo) } \\
\text { Ensino médio } \\
\text { (9-11 anos de estudo) } \\
\text { Ensino superior } \\
\text { (graduação em andamento } \\
\text { ou completa) }\end{array}$ & $\begin{array}{l}61(31,6) \\
85(44,0) \\
44(22,8)\end{array}$ & $\begin{array}{l}27,9 \% \\
20,0 \% \\
13,6 \%\end{array}$ & $0,074^{\dagger}$ \\
\hline $\begin{array}{l}\text { Renda (salários mínimos) } \\
\quad<3 \\
\quad 3 \text { a } 5 \\
>5\end{array}$ & $\begin{array}{l}100(51,8) \\
58(30,1) \\
34(17,6)\end{array}$ & $\begin{array}{l}17,0 \% \\
27,6 \% \\
20,6 \%\end{array}$ & $0,386^{+}$ \\
\hline $\begin{array}{l}\text { Turno de trabalho } \\
\text { Diurno } \\
\text { Noturno }\end{array}$ & $\begin{array}{c}160(82,9) \\
33(17,1)\end{array}$ & $\begin{array}{l}13,1 \% \\
57,6 \%\end{array}$ & $<0,001^{*}$ \\
\hline $\begin{array}{l}\text { Setor de trabalho } \\
\text { Produção } \\
\text { Logística } \\
\text { Administrativo }\end{array}$ & $\begin{array}{l}134(69,4) \\
27(14,0) \\
32(16,6)\end{array}$ & $\begin{array}{c}26,1 \% \\
14,8 \% \\
3,1 \%\end{array}$ & $0,011^{*}$ \\
\hline $\begin{array}{l}\text { Tabagismo } \\
\text { Não } \\
\text { Sim }\end{array}$ & $\begin{array}{c}180(93,3) \\
13(6,7)\end{array}$ & $\begin{array}{l}20,6 \% \\
23,1 \%\end{array}$ & $0,734^{*}$ \\
\hline $\begin{array}{l}\text { Atividade física } \\
\text { Nunca } \\
\leq 3 \text { vezes/semana } \\
>3 \text { vezes/semana }\end{array}$ & $\begin{array}{l}94(48,7 \%) \\
23(11,9 \%) \\
63(32,6 \%)\end{array}$ & $\begin{array}{c}28,7 \% \\
17,4 \% \\
9,5 \%\end{array}$ & $0,0003^{+}$ \\
\hline $\begin{array}{l}\text { Consumo álcool } \\
\text { Nunca } \\
<1 \text { vez/semana } \\
\geq 1 \text { vez/semana }\end{array}$ & $\begin{array}{l}99(51,3 \%) \\
69(35,8 \%) \\
23(11,9 \%)\end{array}$ & $\begin{array}{c}23,2 \% \\
21,7 \% \\
8,7 \%\end{array}$ & $0,298^{+}$ \\
\hline $\begin{array}{l}\text { Remédio para dormir } \\
\text { Não } \\
\text { Sim }\end{array}$ & $\begin{array}{c}181(93,8 \%) \\
11(5,7 \%)\end{array}$ & $\begin{array}{l}19,9 \% \\
36,4 \%\end{array}$ & $0,174^{*}$ \\
\hline
\end{tabular}

* Teste qui-quadrado; ${ }^{\dagger}$ Teste de tendência linear.
Tabela 2. Prevalência de obesidade (índice de massa corporal acima de $30 \mathrm{~kg} / \mathrm{m}^{2}$ ) de acordo com características demográficas, socioeconômicas, ocupacionais e comportamentais de uma população de trabalhadores de uma manufatura do sul do Brasil, entre março e abril, 2015.

\begin{tabular}{|c|c|c|}
\hline Variáveis & $\begin{array}{c}\text { Obesidade } \\
\text { (\%) }\end{array}$ & $\mathbf{p}$ \\
\hline $\begin{array}{l}\text { Sexo } \\
\text { Feminino } \\
\text { Masculino }\end{array}$ & $\begin{array}{l}12,1 \\
16,0\end{array}$ & $0,443 *$ \\
\hline $\begin{array}{l}\text { Idade (quartis) } \\
\qquad 18 \text { a } 25 \text { anos } \\
26 \text { a } 30 \text { anos } \\
31 \text { a } 42 \text { anos } \\
43 \text { a } 60 \text { anos }\end{array}$ & $\begin{array}{c}2,1 \\
16,7 \\
10,0 \\
27,1\end{array}$ & $0,003^{+}$ \\
\hline $\begin{array}{l}\text { Cor de pele } \\
\text { Branco } \\
\text { Não Branco }\end{array}$ & $\begin{array}{l}14,7 \\
10,8\end{array}$ & $0,535^{*}$ \\
\hline $\begin{array}{l}\text { Estado civil } \\
\text { Com companheiro } \\
\text { Sem companheiro }\end{array}$ & $\begin{array}{c}8,6 \\
16,3\end{array}$ & $0,159 *$ \\
\hline $\begin{array}{l}\text { Obesidade parental } \\
\text { Não } \\
\text { Mãe ou pai } \\
\text { Ambos }\end{array}$ & $\begin{array}{c}13,0 \\
8,7 \\
27,8\end{array}$ & $0,128 *$ \\
\hline $\begin{array}{l}\text { Escolaridade } \\
\text { Ensino fundamental } \\
\text { Ensino médio } \\
\text { Ensino superior }\end{array}$ & $\begin{array}{c}26,2 \\
10,6 \\
4,5\end{array}$ & $0,001^{+}$ \\
\hline $\begin{array}{l}\text { Renda (salários mínimos) } \\
\quad \geq 3 \\
3 \text { a } 5 \\
>5\end{array}$ & $\begin{array}{c}13,0 \\
19,0 \\
8,8\end{array}$ & $0,845^{*}$ \\
\hline $\begin{array}{l}\text { Turno de trabalho } \\
\text { Diurno } \\
\text { Noturno }\end{array}$ & $\begin{array}{l}12,5 \\
21,2\end{array}$ & $0,189 *$ \\
\hline $\begin{array}{l}\text { Setor de trabalho } \\
\text { Produção } \\
\text { Logística } \\
\text { Administrativo }\end{array}$ & $\begin{array}{l}17,2 \\
7,4 \\
6,3\end{array}$ & $0,158^{*}$ \\
\hline $\begin{array}{l}\text { Tabagismo } \\
\text { Não } \\
\text { Sim }\end{array}$ & $\begin{array}{l}13,9 \\
15,4\end{array}$ & $0,881^{*}$ \\
\hline $\begin{array}{l}\text { Atividade física } \\
\text { Nunca } \\
\leq 3 \text { vezes/semana } \\
>3 \text { vezes/semana }\end{array}$ & $\begin{array}{l}17,0 \\
13,0 \\
12,7\end{array}$ & $0,729^{+}$ \\
\hline $\begin{array}{l}\text { Consumo álcool } \\
\text { Nunca } \\
<1 \text { vez/semana } \\
\leq 1 \text { vez/semana }\end{array}$ & $\begin{array}{l}15,2 \\
13,0 \\
13,0\end{array}$ & $0,916^{+}$ \\
\hline $\begin{array}{l}\text { Número de refeições/dia } \\
\quad \leq 2 \\
3 \\
\geq 4\end{array}$ & $\begin{array}{c}19,6 \\
15,2 \\
8,8\end{array}$ & $0,098+$ \\
\hline $\begin{array}{l}\text { Duração do sono } \\
\geq 6 \text { horas } \\
<6 \text { horas }\end{array}$ & $\begin{array}{l}10,5 \\
27,5\end{array}$ & $0,006^{*}$ \\
\hline
\end{tabular}

* Teste qui-quadrado; ${ }^{\dagger}$ Teste de tendência linear.

Ensino fundamental, $\leq 8$ anos de estudo; ensino médio, 9-11 anos de estudo; ensino superior, graduação em andamento ou completa. 
Tabela 3. Razões de prevalência ajustadas e intervalos de confiança 95\% para associação entre privação do sono e obesidade de uma população de trabalhadores por turnos de uma manufatura do sul do Brasil. 2015.

\begin{tabular}{|c|c|c|c|c|c|c|c|c|}
\hline \multirow[b]{2}{*}{$\begin{array}{c}\text { RP } \\
(\text { IC95\%) }\end{array}$} & \multicolumn{2}{|c|}{ Modelo 1} & \multicolumn{2}{|c|}{ Modelo 2} & \multicolumn{2}{|c|}{ Modelo 3} & \multicolumn{2}{|c|}{ Modelo 4} \\
\hline & $\begin{array}{c}2,63 \\
(1,22-5,62)\end{array}$ & $0,014^{*}$ & $\begin{array}{c}2,16 \\
(0,95-4,88)\end{array}$ & $0,065^{*}$ & $\begin{array}{c}2,22 \\
(0,94-5,22)\end{array}$ & $0,067^{*}$ & $\begin{array}{c}2,37 \\
(1,08-5,21)\end{array}$ & $0,031^{*}$ \\
\hline
\end{tabular}

RP, razão de prevalência; IC, intervalo de confiança; * p-valor Wald teste.

Regressão de Poisson e teste de Wald para testar a significância de cada variável do modelo.

Modelo 1 (sem ajustes); Modelo 2 (variáveis sociodemográficas); Modelo 3 (modelo 1 + variáveis laborais); Modelo 4 (modelo 1 e 2 + variáveis comportamentais).

\section{DISCUSSÃO}

O principal resultado deste estudo mostra a privação do sono associada à obesidade entre os trabalhadores. Além disso, a ocorrência de privação de sono foi quatro vezes maior entre os trabalhadores do turno noturno com relação aos trabalhadores do turno diurno.

A associação entre privação do sono e obesidade já tem sido relatada na literatura. Em 2008, uma ampla revisão sistemática de artigos publicados entre 1966 e 2007 deu suporte à hipótese de que existe uma associação independente entre duração do sono e ganho de peso ponderal [35]. Um estudo realizado com trabalhadores no Iran observou que o número de horas de sono foi inversamente associado à obesidade, resultado mediado pela ingestão aumentada de energia, carboidratos simples e gorduras [20]. No Brasil, em motoristas de caminhão, foi verificada maior prevalência de obesidade naqueles que dormiam menos de oito horas por dia [36]. Mais recentemente, entre trabalhadores de um frigorífico do sul do Brasil, observou-se que os indivíduos que dormiam menos que seis horas por dia possuíam uma probabilidade quatro vezes maior de apresentar obesidade [16].

Crispim et al. [37] propuseram um modelo teórico no qual a redução do tempo de sono pode modificar padrões endócrinos, que interferem na fome e saciedade. Esse mecanismo seria mediado pelo impacto da privação de sono sobre os reguladores periféricos de saciedade e possível aumento da ingestão calórica total. Estudos têm relacionado a privação do sono com redução nos níveis de leptina e aumento dos níveis de grelina, com consequente aumento do apetite e ganho de peso [21,22,38,39]. Porém, o mecanismo causal que liga a privação de sono à obesidade ainda não está completamente elucidado. Pesquisas também têm indicado que a privação do sono prolongada, associada à disrupção do ritmo circadiano, pode afetar a tolerância à glicose $[24,40,41]$. Outra questão ainda sem conseso na literatura é a definição de privação de sono. As definições de duração de sono normal variam entre 6 a 9 horas por dia [35], porém distúrbios metabólicos e ganho de peso podem ser observados em indivíduos que dormem menos do que 6 horas por dia [42-44].

A obesidade é um agravo multifatorial. Por isso, neste estudo, a associação entre privação do sono e obesidade foi controlada para fatores sociodemográficos, ocupacionais e comportamentais por meio de análise multivariada de dados. Ao controlar o modelo para as características sociodemográficas (idade, estado civil e escolaridade) e obesidade parental, a associação entre privação do sono e obesidade perdeu significância estatística. Porém, ao se inserir a variável comportamental, número de refeições, a associação voltou a ser significativa. Essas variáveis são conhecidos fatores associados à obesidade [45,46], e mostraram-se importantes fatores de confusão neste estudo, reforçando a importância da sua inclusão em estudos que investigem os fatores associados à obesidade.

O turno de trabalho não esteve associado à obesidade neste estudo. Porém, trabalhar no turno noturno foi associado positivamente à privação de sono pela análise bivariada. A privação do sono parece ser uma consequência imediata do trabalho noturno, uma vez que se está trabalhando no horário reservado ao sono. Em um estudo realizado com 905 trabalhadores de um frigorífico de frango no sul do Brasil, todos os trabalhadores expostos à privação de sono eram trabalhadores do turno noturno [15]. Dessa forma, o trabalho noturno pode ser um fator de risco para obesidade, tendo como fator mediador a privação do sono. É difícil distinguir os papéis da privação do sono e do trabalho noturno no ganho excessivo de peso. Da mesma forma que a privação do sono leva a diversas alterações metabólicas, o relógio biológico está programado para responder às flutuações naturais na temperatura e luz ambiente durante as 24 horas do dia. A ampla utilização de meios artificiais de luminosidade, como é o caso do trabalho noturno, pode diminuir a sincronização do ritmo circadiano e aumentar o risco de desenvolvimento de desajustes entre o ambiente natural e o relógio biológico [47]. 
Estes desalinhamentos podem levar a alterações no metabolismo e na termorregulação que promovem a obesidade $[48,49]$.

Neste estudo, a privação do sono esteve associada ao setor de produção. Esse achado é explicado pelo fato de que grande maioria dos trabalhadores desse setor trabalhar no turno noturno. Os setores de logística e administrativo funcionam apenas no turno diurno.

Não praticar atividade física foi outra característica associada à privação do sono entre os trabalhadores. Pode-se supor que o sedentarismo tenha levado à má qualidade do sono. Porém, por tratar-se de um estudo transversal, passível de viés de causalidade reversa, parece mais plausível que a privação do sono tenha limitado a prática de atividades físicas de lazer como consequência do cansaço.

Os resultados deste estudo devem ser interpretados no contexto de algumas limitações. As medidas de peso e altura foram autorreferidas, porém, recentemente, os trabalhadores passaram por uma avaliação antropométrica e receberam o resultado por escrito. Isso pode ter aumentado a acurácia dos dados por eles fornecidos. Além disso, diversos estudos têm feito uso de medidas autorreferidas com população em geral, como é o caso do VIGITEL, e com trabalhadores [50,51], demonstrado a sua validade. Trata-se de um estudo transversal onde a doença e a exposição foram avaliadas concomitantemente, o que dificulta avaliar se a privação de sono levou a obesidade ou a obesidade induziu a distúrbios de sono. Houve uma perda amostral importante no processo da pesquisa (em torno de $25 \%$ ), o que pode afetar a validade externa dos resultados. Além disso, os resultados deste estudo referem-se a trabalhadores alocados em turnos fixos e não podem ser extrapolados para turnos rotativos.

Este estudo demostrou que trabalhadores em privação do sono têm maior probabilidade de desenvolver obesidade. Além disso, o trabalho noturno parece desempenhar um importante papel nessa associação, uma vez que os trabalhadores do turno noturno apresentaram maior prevalência de privação de sono. Futuros estudos que investiguem os mecanismos de ação da privação de sono na obesidade e o papel do trabalho em turnos nessa relação devem ser conduzidos, em diferentes populações e com diferentes desenhos metodológicos, aumentado o corpo de evidências que baseiam as estratégias de melhora da qualidade de vida de trabalhadores.

\section{NOTA}

Declaração de conflitos de interesse

Os autores declaram não haver conflitos de interesse relevantes ao conteúdo deste estudo.

\section{REFERÊNCIAS}

1. Finucane MM, Stevens GA, Cowan MJ, Danaei G, Lin JK, Paciorek CJ, Singh GM, Gutierrez HR, Lu Y, Bahalim AN, Farzadfar F, Riley LM, Ezzati M; Global Burden of Metabolic Risk Factors of Chronic Diseases Collaborating Group (Body Mass Index). National, regional, and global trends in bodymass index since 1980: systematic analysis of health examination surveys and epidemiological studies with 960 country-years and 9.1 million participants. Lancet. 2011 Feb 12;377(9765): 557-67. http://dx.doi.org/10.1016/S0140-6736(10)62037-5

2. Guh DP, Zhang W, Bansback N, Amarsi Z, Birmingham CL, Anis AH. The incidence of co-morbidities related to obesity and overweight: a systematic review and meta-analysis. BMC Public Health. 2009 Mar 25;9:88. http://dx.doi.org/10.1186/14712458-9-88

3. Global Burden of Metabolic Risk Factors for Chronic Diseases Collaboration. Cardiovascular disease, chronic kidney disease, and diabetes mortality burden of cardiometabolic risk factors from 1980 to 2010: a comparative risk assessment. Lancet Diabetes Endocrinol. 2014 Aug;2(8):634-47. http://dx.doi.org/10.1016/S2213-8587(14)70102-0

4. Wolk R, Shamsuzzaman ASM, Somers, VK. Obesity, Sleep Apnea, and Hypertension. Hypertension. 2003 Dec;42(6): 1067-74. http://dx.doi.org/10.1161/01.HYP.0000101686.98973.A3

5. Garrison RJ, Kannel WB, Stokes J III, Castelli WP. Incidence and precursors of hypertension in young adults: the Framingham Offspring Study. Prev Med. 1987 Mar;16(2):235-51. http://dx.doi.org/10.1016/0091-7435(87)90087-9

6. Young T, Peppard PE, Gottlieb DJ. Epidemiology of obstructive sleep apnea: a population health perspective. Am J Respir Crit Care Med. 2002 May 1;165(9):1217-39. http://dx.doi.org/10.1164/rccm.2109080

7. Danaei G, Finucane MM, Lu Y, Singh GM, Cowan MJ, Paciorek CJ, Lin JK, Farzadfar F, Khang YH, Stevens GA, Rao M, Ali MK, Riley LM, Robinson CA, Ezzati M. Global Burden of Metabolic Risk Factors of Chronic Diseases Collaborating Group (Blood Glucose). National, regional, and global trends in fasting plasma glucose and diabetes prevalence since 1980: systematic analysis of health examination surveys and epidemiological studies with 370 country-years and $2 \cdot 7$ million participants. Lancet. 2011 Jul 2;378(9785):31-40. http://dx.doi.org/10.1016/S0140-6736(11)60679-X 
8. Sattar N, Gill JMR. Type 2 diabetes as a disease of ectopic fat? BMC Med. 2014 Aug 26;12:123. http://dx.doi.org/10.1186/ s12916-014-0123-4

9. De Pergola G, Silvestris F. Obesity as a major risk factor for cancer. J Obes. 2013;2013:291546. http://dx.doi. org/10.1155/2013/291546

10. Strazzullo P, D’Elia L, Cairella G, Garbagnati F, Cappuccio FP, Scalfi L. Excess body weight and incidence of stroke: meta-analysis of prospective studies with 2 million participants. Stroke. 2010 May;41(5):e418-26. http://dx.doi.org/10.1161/ STROKEAHA.109.576967

11. Global Burden of Metabolic Risk Factors for Chronic Diseases Collaboration (BMI Mediated Effects), Lu Y, Hajifathalian K, Ezzati M, Woodward M, Rimm EB, Danaei G. Metabolic mediators of the effects of body-mass index, overweight, and obesity on coronary heart disease and stroke: a pooled analysis of 97 prospective cohorts with 1.8 million participants. Lancet. 2014 Mar 15;383(9921):970-83. http://dx.doi.org/10.1016/S0140-6736(13)61836-X

12. Brasil. Secretaria de Vigilância em Saúde. Vigitel Brasil 2014: vigilância de fatores de risco e proteção para doenças crônicas por inquérito telefônico. Brasília: Ministério da Saúde; 2015.

13. Olinto MTA; Canuto R; da Silva Garcez A. Work and abdominal obesity risk. In: Ronald Ross Watson, organizators. nutrition in the prevention and treatment of abdominal obesity. Cambridge: Academic Press; 2014. p. 17-25. http://dx.doi.org/10.1016/ B978-0-12-407869-7.00002-7

14. Crispim CB, Waterhouse J, Damaso AR, Zimberg IZ, Padilha HG, Oyama LM, Tufik S, Mello MT. Hormonal appetite control is altered by shift work: a preliminary study. Metabolism. 2011 Dec;60(12):1726-35. http://dx.doi.org/10.1016/j. metabol.2011.04.014

15. Macagnan J, Pattussi MP, Canuto R, Henn RL, Fassa AG, Olinto MT. Impact of nightshift work on overweight and abdominal obesity among workers of a poultry processing plant in southern Brazil. Chronobiol Int. 2012 Apr;29(3):336-43. http://dx.doi. org/10.3109/07420528.2011.653851

16. Canuto R, Patussi MP, Macagnan JBA, Henn RL, Olinto MTA. Sleep deprivation and obesity in shift workers in southern Brazil. Public Health Nutr. 2014 Nov;17(11):2619-23. http://dx.doi.org/10.1017/S1368980013002838

17. Eberly R, Feldman H. Obesity and shift work in the general population. IJAHSP. 2010;8(3):1-9.

18. Geliebter A, Gluck ME, Tanowitz M, Aronoff NJ, Zammit GK. Work-shift period and weight change. Nutrition. 2000 Jan;16(1):27-9. http://dx.doi.org/10.1016/S0899-9007(99)00228-2

19. Reeves SL, Newling-Ward E, Gissane C. The effect of shift-work on food intake and eating habits. Nutr Food Sci. 2004;34(5):216-21. http://dx.doi.org/10.1108/00346650410560398

20. Parvaneh K, Poh BK, Hajifaraji M, Ismail MN. Sleep deprivation is related to obesity and low intake of energy and carbohydrates among working Iranian adults: a cross sectional study. Asia Pac J Clin Nutr. 2014;23(1):84-90.

21. Davies SK, Ang JE, Revell VL, Holmes B, Mann A, Robertson FP, Cui N, Middleton B, Ackermannc K, Kayser M, Thumser AE, Raynaudb FI, Skene DJ. Effect of sleep deprivation on the human metabolome. Proc Natl Acad Sci U S A. 2014 Jul 22;111(29):10761-6. http://dx.doi.org/10.1073/pnas.1402663111

22. Bass J, Takahashi JS. Circadian integration of metabolism and energetics. Science. 2010 Dec 3;330(6009):1349-54. http:// dx.doi.org/10.1126/science.1195027

23. Spiegel K, Tasali E, Penev P, Van Cauter E. Brief communication: Sleep curtailment in healthy young men is associated with decreased leptin levels, elevated ghrelin levels, and increased hunger and appetite. Ann Intern Med. 2004 Dec 7;141(11): 846-50. http://dx.doi.org/10.7326/0003-4819-141-11-200412070-00008

24. Spiegel K, Knutson K, Leproult R, Tasali E, Van Cauter E. Sleep loss: A novel risk factor for insulin resistance and type 2 diabetes. J Appl Physiol (1985). 2005 Nov;99(5):2008-19.

25. Bass J, Turek FW. Sleepless in America: a pathway to obesity and the metabolic syndrome? Arch Intern Med. 2005 Jan 10;165(1):15-6. http://dx.doi.org/10.1001/archinte.165.1.15

26. Canuto R, Garcez AS, Olinto, MTA. Metabolic syndrome and shift work: A systematic review. Sleep Med Rev. 2013 Dec;17(6):425-31 http://dx.doi.org/10.1016/j.smrv.2012.10.004

27. Kerkhofs M, Boudjeltia KZ, Stenuit P, Brohee D, Cauchie P, Vanhaeverbeek M. Sleep restriction increases blood neutrophils, total cholesterol and low density lipoprotein cholesterol in postmenopausal women: a preliminary study. Maturitas. 2007 Feb 20;56(2):212-5. http://dx.doi.org/10.1016/j.maturitas.2006.07.007

28. Poulsen K, Cleal B, Clausen T, Andersen LL. Work, diabetes and obesity: A seven year follow-up study among Danish Health care workers. PLoS One. 2014 Jul 28;9(7):e103425. http://dx.doi.org/10.1371/journal.pone.0103425

29. Canuto, R, Patussi, MP, Macagnan JBA, Henn RL, Olinto MTA. Metabolic syndrome in fixed-shift workers. Rev. Saúde Públ. 2015;49(30):1-8. http://dx.doi.org/10.1590/S0034-8910.2015049005524

30. Scheer FAJL, Hilton MF, Mantzoros CS, Shea SA. Adverse metabolic and cardiovascular consequences of circadian misalignment. Proc Natl Acad Sci U S A. 2009 Mar 17;106(11):4453-8. http://dx.doi.org/10.1073/pnas.0808180106

31. Tüchsen F, Hannerz H, Burr H. A 12 year prospective study of circulatory disease among Danish shift workers. Occup Environ Med. 2006 Jul;63(7):451-5. http://dx.doi.org/10.1136/oem.2006.026716 
32. Esquirol Y, Perret B, Ruidavets JB, Marquie JC, Dienne E, Niezborala M, Ferrieres J. Shift work and cardiovascular risk factors: new knownledge from the past decade. Arch Cardiovasc Dis. 2011 Dec;104(12):636-68. http://dx.doi.org/10.1016/j. acvd.2011.09.004

33. Cappuccio FP, Cooper D, D’Elia L, Strazzullo P, Miller MA. Sleep duration predicts cardiovascular outcomes: a systematic review and meta-analysis of prospective studies. Eur Heart J. 2011 Jun;32(12):1484-92. http://dx.doi.org/10.1093/eurheartj/ ehr007

34. World Health Organization. Obesity: preventing and managing the global epidemic. Geneva: WHO; 2000. WHO Technical Report Series, 894.

35. Patel SR, Hu FB. Short sleep duration and weight gain: a systematic review. Obesity (Silver Spring). 2008 Mar;16(3):64353. http://dx.doi.org/10.1038/oby.2007.118

36. Moreno CR, Louzada FM, Teixeira LR, Borges F, Lorenzi-Filho G. Short sleep is associated with obesity among truck drivers. Chronobiol Int. 2006;23(6):1295-303. http://dx.doi.org/10.1080/07420520601089521

37. Crispim CA, Zalcman I, Datillo M, Padilha HG, Tufik S, Mello MT. Relação entre sono e obesidade: uma revisão da literatura. Arq Bras Endocrinol Metabol. 2007 Oct;51(7):1041-9. http://dx.doi.org/10.1590/S0004-27302007000700004

38. Shea SA, Hilton MF, Orlova C, Ayers RT, Mantzoros CS. Independent circadian and sleep/wake regulation of adipokines and glucose in humans. J Clin Endocrinol Metab. 2005 May;90(5):2537-44. http://dx.doi.org/10.1210/jc.2004-2232

39. Yildiz BO, Suchard MA, Wong ML, McCann SM, Licinio J. Alterations in the dynamics of circulating ghrelin, adiponectin, and leptin in human obesity. Proc Natl Acad Sci U S A. 2004 Jul 13;101(28):10434-9. http://dx.doi.org/10.1073/pnas.0403465101

40. Kalsbeek A, La Fleur S, Fliers E. Circadian control of glucose metabolism. Mol Metab. 2014 Mar 19;3(4):372-83. http:// dx.doi.org/10.1016/j.molmet.2014.03.002

41. Buxton OM, Cain SW, O’Connor SP, Porter JH, Duffy JF, Wang W, Czeisler CA, Shea SA. Adverse metabolic consequences in humans of prolonged sleep restriction combined with circadian disruption. Sci Transl Med. 2012 Apr 11;4(129):129ra43. http://dx.doi.org/10.1126/scitranslmed.3003200

42. Benedict C, Hallschmid M, Lassen A, Mahnke C, Schultes B, Schiöth HB, Born J, Lange T. Acute sleep deprivation reduces energy expenditure in healthy men. Am J Clin Nutr. 2011 Jun;93(6):1229-36. http://dx.doi.org/10.3945/ajcn.110.006460

43. Knutson KL, Spiegel K, Penev P, Van Cauter E. The metabolic consequences of sleep deprivation. Sleep Med Rev. 2007 Jun;11(3):163-78. http://dx.doi.org/10.1016/j.smrv.2007.01.002

44. Chaput JP, Despres JP, Bouchard C, Tremblay A. Short sleep duration is associated with reduced leptin levels and increased adiposity: results from the Quebec Family Study. Obesity (Silver Spring). 2007 Jan;15(1):253-61. http://dx.doi.org/10.1038/ oby.2007.512

45. Malta DC, Moehlecke Iser BP, Claro RM, Moura L, Bernal RTI, Nascimento AF, Silva JBJ, Monteiro CA. Prevalência de fatores de risco e proteção para doenças crônicas não transmissíveis em adultos residentes em capitais brasileiras. Epidemiol Serv Saúde. 2013;22(3):423-34. http://dx.doi.org/10.5123/S1679-49742013000300007

46. .Murakami K, Livingstone MB. Eating Frequency Is Positively Associated with Overweight and Central Obesity in U.S. Adults. J Nutr. 2015 Dec;145(12):2715-24. http://dx.doi.org/10.3945/jn.115.219808

47. Kanikowska D, Sato M, Witowski J. Contribution of daily and seasonal biorhythms to obesity in humans. Int J Biometeorol. 2015 Apr;59(4):377-84. http://dx.doi.org/10.1007/s00484-014-0871-z

48. Johnson F, Mavrogianni A, Ucci M, Vidal-Puig A, Wardle J. Could increased time spent in a thermal comfort zone contribute to population increases in obesity? Obes Rev. 2011 Jul;12(7):543-51. http://dx.doi.org/10.1111/j.1467-789X.2010.00851.x

49. Wyse CA, Selman C, Page MM, Coogan AN, Hazlerigg DG. Circadian desynchrony and metabolic dysfunction; did light pollution make us fat? Med Hypotheses. 2011 Dec;77(6):1139-44. http://dx.doi.org/10.1016/j.mehy.2011.09.023

50. Dekkers JC, van Wier MF, Hendriksen IJ, Twisk JW, van Mechelen W. Accuracy of self-reported body weight, height and waist circumference in a Dutch overweight working population. BMC Med Res Methodol. 2008 Oct 28;8:69. http://dx.doi. org/10.1186/1471-2288-8-69

51. Wada K, Tamakoshi K, Tsunekawa T, Otsuka R, Zhang H, Murata C, Nagasawa N, Matsushita K, Sugiura K, Yatsuya H, Toyoshima H. Validity of self-reported height and weight in a Japanese workplace population. Int J Obes (Lond). 2005 Sept;29(9):1093-9. http://dx.doi.org/10.1038/sj.ijo.0803012 